\title{
Erratum to: Information on the inflaton field from the spectrum of relic gravitational waves
}

\section{Christian Corda}

Published online: 8 December 2009

(C) Springer Science+Business Media, LLC 2009

\section{Erratum to: Gen Relativ Gravit DOI 10.1007/s10714-009-0895-6}

In Eqs. (22) and (25) of the paper "Information on the inflaton field from the spectrum of relic gravitational waves," DOI 10.1007/s10714-009-0895-6, integral signs are missing.

Thus, Eq. (22) has to be substituted with

$$
S=\int d^{4} x \sqrt{-g}\left[\varphi R-\frac{\omega(\varphi)}{\varphi} g^{\mu \nu} \varphi_{; \mu} \varphi_{; \nu}-W(\varphi)+\mathcal{L}_{m}\right],
$$

while Eq. (25) has to be substituted with

$$
A=\int \frac{1}{2 k} d^{4} x \sqrt{-\widetilde{g}}\left[\widetilde{R}+L\left(\Phi, \Phi_{; \alpha}\right)\right]
$$

A typo is also present in Ref. [18]: the year should be 1981, not 1982.

My apologies to the Scientific Community for such typos.

Some historical notes: Eq. (17) first appeared in Ref. [6], and in a more rigorous form which remains valid after the end of the RD stage - with $\rho_{\text {rad }}$ instead of $\rho_{c}$ where $\rho_{\text {rad }}$ is the total radiation energy density at the moment of the second Hubble radius crossing at the RD stage, see Eq. (6) in Ref. [6].

The online version of the original article can be found under doi:10.1007/s10714-009-0895-6.

C. Corda $(\bowtie)$

Associazione Scientifica Galileo Galilei,

Via Pier Cironi 16, 59100 Prato, Italy

e-mail: cordac.galilei@gmail.com 
An analytical calculation of the contribution from GW to the Sachs-Wolfe plateau was in the paper by A. A. Starobinsky, Sov. Astron. Lett. 11, 133 (1985) which has to be added to Ref. [18] for a sake of completeness.

I strongly thank Professor A. A. Starobinsky for important clarifications on historical notes concerning this issue. 